\title{
Linear-Chain AlPOs Obtained by the Reaction of Amine Phosphates with $\mathrm{Al}^{3+}$ lons
}

\author{
Ayi A. Ayi, ${ }^{*, 1}$ Amitava Choudhury, ${ }^{*} \dagger$ and Srinivasan Natarajan ${ }^{*, 2}$ \\ *Chemistry and Physics of Materials Unit, Jawaharlal Nehru Centre for Advanced Scientific Research, Jakkur P.O., Bangalore 560064, India; and \\ $\dagger$ Solid State and Structural Chemistry Unit, Indian Institute of Science, Bangalore 560012 , India
}

Received June 14, 2000; in revised form August 29, 2000; accepted October 3, 2000; published online December 21, 2000

Three one-dimensional aluminum phosphates $\left[\mathrm{H}_{3} \mathrm{~N}\left(\mathrm{CH}_{2}\right)_{3}\right.$ $\left.\mathrm{NH}_{3}\right]\left[\mathrm{Al}\left(\mathrm{HPO}_{4}\right)\left(\mathrm{PO}_{4}\right)\right], \mathrm{I},\left[\mathrm{H}_{3} \mathrm{NCH}_{2} \mathrm{CH}\left(\mathrm{NH}_{3}\right) \mathrm{CH}_{3}\right]\left[\mathrm{NH}_{4}\right]$ $\left[\mathrm{Al}\left(\mathrm{PO}_{4}\right)_{2}\right], \mathrm{II},\left[\mathrm{H}_{3} \mathrm{~N}\left(\mathrm{CH}_{2}\right)_{3} \mathrm{NH}_{3}\right]\left[\mathrm{N}_{2} \mathrm{H}_{6} \mathrm{O}_{2}\right]_{0.5}\left[\mathrm{Al}\left(\mathrm{PO}_{4}\right)_{2}\right], \mathrm{III}$, containing linear chains of corner-shared four-membered rings have been synthesized by the reaction between amine phosphates and $\mathrm{Al}^{3+}$ ions. The structures consist of macroanionic $\left[\mathrm{Al}\left(\mathrm{PO}_{4}\right)\left(\mathrm{HPO}_{4}\right)\right]^{2-}$ or $\left[\mathrm{Al}\left(\mathrm{PO}_{4}\right)_{2}\right]^{3-}$ chains built up from $\mathrm{AlO}_{4}$ and $\mathrm{PO}_{4}$ tetrahedra, in which all the $\mathrm{AlO}_{4}$ vertices are shared but each $\mathrm{PO}_{4}$ has two terminal $\mathrm{P}-\mathrm{O}$ linkages. The framework negative charge is balanced by the amine related cations holding phosphate chains together through hydrogen bond interactions. The chain with the composition $\left[\mathrm{Al}\left(\mathrm{PO}_{4}\right)\left(\mathrm{HPO}_{4}\right)\right]$ has been observed for the first time. (1) 2001 Academic Press

\section{INTRODUCTION}

After the seminal work by Wilson et al. (1), a large number of aluminophosphates (AlPO- $n$ with $n$ representing a particular structure type) have been reported encompassing a wide range of structure types both zeolitic and novel. Structurally, most AlPO- $n$ phases are exclusively composed of $\mathrm{AlO}_{4}$ and $\mathrm{PO}_{4}$ primary building units although a few contain $\mathrm{AlO}_{5}$ and $\mathrm{AlO}_{6}$ units $(2,3)$. Of the many AlPOs, structures with three-dimensional structures, e.g., AlPO-5 (SSZ-23) (1) and AlPO-34 (chabazite) (1), and two-dimensional structures, e.g., $\left[\mathrm{NH}_{3}\left(\mathrm{CH}_{2}\right)_{4} \mathrm{NH}_{3}\right]_{1.5}\left[\mathrm{Al}_{3}\left(\mathrm{PO}_{4}\right)_{4}\right]$ (4) and $\left[\mathrm{NH}_{3}\left(\mathrm{CH}_{2}\right)_{2} \mathrm{NH}_{3}\right]\left[\mathrm{Al}_{2}(\mathrm{OH})_{2} \mathrm{H}_{2} \mathrm{O}\left(\mathrm{PO}_{4}\right)_{2}\right] \mathrm{H}_{2} \mathrm{O}(5)$ are more common than the one-dimensional ones. The onedimensional AlPOs exist in two distinct forms, an edgeshared ladder $(6,7)$ and a corner-shared chain $(8,9)$. Of these, the ladders are prominent. The ladder structures contain a backbone of edge-shared four-membered rings and possess additional pendant $\mathrm{H}_{2} \mathrm{PO}_{4}$ or $\mathrm{HPO}_{4}$ units. The

\footnotetext{
${ }^{1}$ Permanent address: Department of Chemistry, University of Calabar. P.M.B. 11115 - Calabar, Nigeria.

${ }^{2}$ To whom correspondence should be addressed. E-mail: raj@ jncasr.ac.in: Fax: (+)080-846-2766.
}

chains, on the other hand, are made from a corner sharing of $\mathrm{AlO}_{4}$ and $\mathrm{PO}_{4}$ tetrahedra and contain no pendant groups, the chain compositions being $\left[\mathrm{Al}\left(\mathrm{PO}_{4}\right)_{2}\right]^{3-}(8 \mathrm{a})$, $\left[\mathrm{Al}\left(\mathrm{HPO}_{4}\right)_{2}\right]^{-}(8 \mathrm{~b})$. Recently it has been shown that the reaction of metal ions with amine phosphates offers a facile route for the synthesis of open-framework materials (10). Using this method, a layered AlPO possessing tancoite-type structure (11) has been synthesized (5). In continuation of the theme, we have now isolated and characterized three one-dimensional AlPOs of the composition, $\left[\mathrm{H}_{3} \mathrm{~N}\left(\mathrm{CH}_{2}\right)_{3} \mathrm{NH}_{3}\right]\left[\mathrm{Al}\left(\mathrm{HPO}_{4}\right)\left(\mathrm{PO}_{4}\right)\right], \quad \mathbf{I}, \quad\left[\mathrm{H}_{3} \mathrm{NCH}_{2} \mathrm{CH}\right.$ $\left.\left(\mathrm{NH}_{3}\right) \mathrm{CH}_{3}\right] \quad\left[\mathrm{NH}_{4}\right]\left[\mathrm{Al}\left(\mathrm{PO}_{4}\right)_{2}\right]$, II, $\left[\mathrm{H}_{3} \mathrm{~N}\left(\mathrm{CH}_{2}\right)_{3} \mathrm{NH}_{3}\right]$ $\left[\mathrm{N}_{2} \mathrm{H}_{6} \mathrm{O}_{2}\right]_{0.5}\left[\mathrm{Al}\left(\mathrm{PO}_{4}\right)_{2}\right]$, III, by the reaction of $\mathrm{Al}^{3+}$ ions with amine phosphates. The solids form into two distinct types possessing the chain compositions of $\left[\mathrm{Al}\left(\mathrm{PO}_{4}\right)\left(\mathrm{HPO}_{4}\right)\right]^{2-}$ and $\left[\mathrm{Al}\left(\mathrm{PO}_{4}\right)_{2}\right]^{3-}$. The chain composition, $\left[\mathrm{Al}\left(\mathrm{PO}_{4}\right)\left(\mathrm{HPO}_{4}\right)\right]^{2-}$, has been observed for the first time, though the same composition is known in gallium phosphate chains (12). In this paper we present the synthesis and structures of the one-dimensional phosphates which are rather uncommon compared to the two- and three-dimensional phosphate structures.

\section{EXPERIMENTAL}

The compounds I-III were synthesized by the reaction of $\mathrm{Al}^{3+}$ ions with 1,2-diammoniumpropane phosphate (1,2DAP-P) and 1,3-diammoniumpropane phosphate (1,3DAP-P). These amine phosphates were prepared by standard procedure (13). While the structure of 1,3-DAP-P is known (13), that of 1,2-DAP-P was established in this study.

In a typical synthesis as in the case of $\mathbf{I}, 0.117 \mathrm{~g}$ of $\mathrm{Al}(\mathrm{OH})_{3}$ was dispersed in $0.54 \mathrm{ml}$ of water, followed by the addition of $0.14 \mathrm{ml}$ of dioxane and $0.25 \mathrm{ml}$ of $\mathrm{HCl}$. Finally, $0.856 \mathrm{~g}$ of 1,3-DAP-P was added to above under constant stirring to result in a homogeneous gel. The gel was transferred, sealed in a PTFE-lined stainless steel acid digestion bombs and heated at $180^{\circ} \mathrm{C}$ for $48 \mathrm{~h}$. The resulting product, a crop of fine needles, was vacuum filtered and dried at 
TABLE 1

Synthesis Conditions ${ }^{a}$ and Analysis for Compounds I-III

\begin{tabular}{|c|c|c|c|}
\hline \multirow[b]{2}{*}{ Mole ratio } & \multicolumn{2}{|r|}{ Analysis } & \multirow[b]{2}{*}{ Composition } \\
\hline & $\begin{array}{l}\text { EDAX } \\
(\mathrm{Al} / \mathrm{P})\end{array}$ & TGA (\% wt. loss $)^{b}$ & \\
\hline $\begin{array}{l}\mathrm{Al}(\mathrm{OH})_{3}: 2 \mathrm{HCl}: 3(1,3 \\
\mathrm{DAPP}): 20 \mathrm{H}_{2} \mathrm{O}:\end{array}$ & & & \\
\hline Dioxane & $1: 2$ & 26.1(28.9) & $\begin{array}{c}{\left[\mathrm{C}_{3} \mathrm{~N}_{2} \mathrm{H}_{12}\right]\left[\mathrm{Al}\left(\mathrm{HPO}_{4}\right)\right.} \\
\left.\left(\mathrm{PO}_{4}\right)\right], \mathbf{I}\end{array}$ \\
\hline $\mathrm{Al}(\mathrm{OH})_{3}:(1,2$ DAPP $)$ & & & \\
\hline $\mathrm{N}_{2} \mathrm{H}_{4} \cdot 2 \mathrm{H}_{2} \mathrm{O}: 20 \mathrm{H}_{2} \mathrm{O}$ & $1: 2$ & $26.2(302)$ & $\begin{array}{c}{\left[\mathrm{C}_{3} \mathrm{~N}_{2} \mathrm{H}_{12}\right]} \\
\left.\mathrm{Al}\left(\mathrm{PO}_{4}\right)_{2}\right],\end{array}$ \\
\hline $\begin{array}{l}\mathrm{Al}(\mathrm{OH})_{3}:(1,3 \text { DAPP }): \\
\mathrm{N}_{2} \mathrm{H}_{4} \cdot 2 \mathrm{H}_{2} \mathrm{O}: 20 \mathrm{H}_{2} \mathrm{O}\end{array}$ & $1: 2$ & $23.2(28.4)$ & $\begin{array}{c}{\left[\mathrm{C}_{3} \mathrm{~N}_{2} \mathrm{H}_{12}\right]\left[\mathrm{N}_{2} \mathrm{O}_{2} \mathrm{H}_{6}\right]_{0.5}} \\
{\left[\mathrm{Al}\left(\mathrm{PO}_{4}\right)_{2}\right], \text { IIII }}\end{array}$ \\
\hline
\end{tabular}

${ }^{a}$ The typical amount of $\mathrm{Al}(\mathrm{OH})_{3}$ is $1.5 \mathrm{mM}$. All the reaction mixture was heated at $453 \mathrm{~K}$ for $48 \mathrm{~h}$.

${ }^{b}$ Values in the parenthesis are calculated value. For I the mass loss includes phosphoryl-OH.

ambient conditions. Hydrazine hydrate was taken in the starting mixture along with 1,2-DAP-P and 1,3-DAP-P for the synthesis of II and III, respectively. The gel composition,

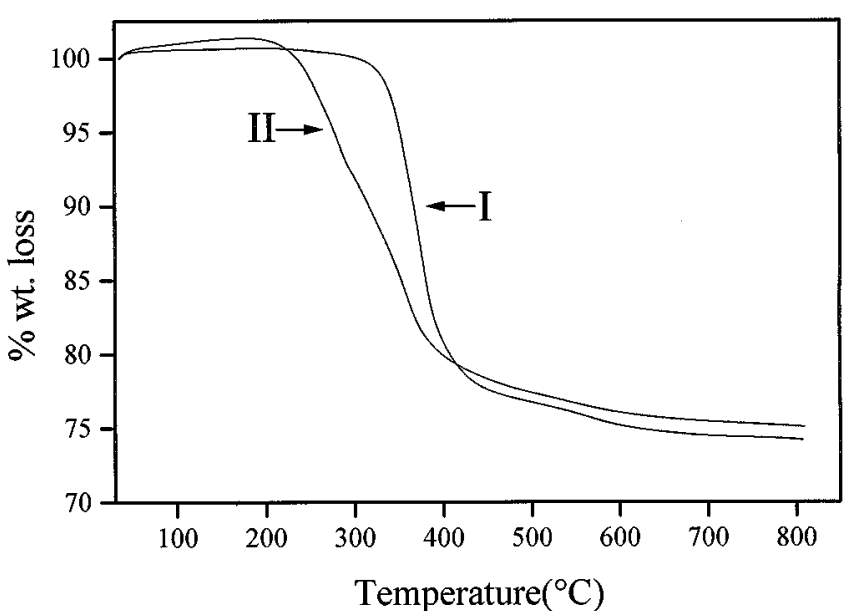

FIG. 1. TGA curves for compounds I and II.

synthetic conditions, and product compositions are listed in Table 1 . The products were characterized by single crystal $\mathrm{X}$-ray diffraction and thermogravimetric analysis (TGA).

TGA studies were carried out in $\mathrm{N}_{2}$ atmosphere (flow rate $=50 \mathrm{ml} / \mathrm{min}$ ) in the range room temperature to $700^{\circ} \mathrm{C}$

TABLE 2

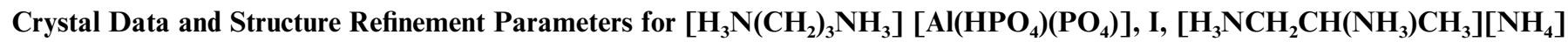
$\left[\mathrm{Al}\left(\mathrm{PO}_{4}\right)_{2}\right]$, II, and $\left[\mathrm{H}_{3} \mathbf{N}\left(\mathrm{CH}_{2}\right)_{3} \mathbf{N H}_{3}\right]\left[\mathrm{N}_{2} \mathrm{H}_{6} \mathrm{O}_{2}\right]_{0.5}\left[\mathrm{Al}\left(\mathrm{PO}_{4}\right)_{2}\right]$, III

\begin{tabular}{|c|c|c|c|}
\hline Structural parameter & $\mathbf{I}$ & II & III \\
\hline Empirical formula & $\mathrm{AlP}_{2} \mathrm{O}_{8} \mathrm{C}_{3} \mathrm{~N}_{2} \mathrm{H}_{13}$ & $\mathrm{AlP}_{2} \mathrm{O}_{8} \mathrm{C}_{3} \mathrm{~N}_{3} \mathrm{H}_{16}$ & $\mathrm{AlP}_{2} \mathrm{O}_{9} \mathrm{C}_{3} \mathrm{~N}_{3} \mathrm{H}_{15}$ \\
\hline Crystal system & Triclinic & Orthorhombic & Triclinic \\
\hline Space group & $P(-1)$ (No. 2) & Pccn (No. 56) & $P(-1)$ (No. 2) \\
\hline$a(\AA)$ & $8.309(1)$ & $16.832(2)$ & $8.669(1)$ \\
\hline$b(\AA)$ & $8.636(1)$ & $8.289(3)$ & $8.943(1)$ \\
\hline$c(\AA)$ & $8.844(1)$ & $8.694(2)$ & $9.266(1)$ \\
\hline$\alpha$ & 111.9(1) & 90.0 & $98.3(1)$ \\
\hline$\beta\left(^{\circ}\right)$ & 107.6(1) & 90.0 & $116.0(2)$ \\
\hline$\gamma$ & $98.0(1)$ & 90.0 & $99.7(1)$ \\
\hline Volume $\left(\AA^{3}\right)$ & $537.6(1)$ & 1212.1(3) & $616.9(1)$ \\
\hline$Z$ & 2 & 4 & 2 \\
\hline Formula mass & 294.07 & 312.24 & 326.1 \\
\hline$\rho_{\text {calc }}\left(\mathrm{g} \mathrm{cm}^{-3}\right)$ & 1.817 & 1.710 & 1.756 \\
\hline$\mu\left(\mathrm{mm}^{-1}\right)$ & 0.517 & 0.470 & 0.468 \\
\hline$\theta$ Range $\left({ }^{\circ}\right)$ & $2.65-23.32$ & $2.42-23.25$ & $2.38-23.29$ \\
\hline Total data collected & 2292 & 4514 & 2638 \\
\hline Index ranges & $\begin{array}{l}-8 \leq h \leq 9, \quad-7 \leq k \leq 9 \\
-9 \leq l \leq 9\end{array}$ & $\begin{array}{l}-12 \leq h \leq 18, \quad-8 \leq k \leq 9 \\
-9 \leq l \leq 9\end{array}$ & $\begin{array}{l}-9 \leq h \leq 9,-9 \leq k \leq 9 \\
-10 \leq l \leq 6\end{array}$ \\
\hline Unique data & 1526 & 873 & 1760 \\
\hline $\operatorname{Data}[I>2 \sigma(I)]$ & 1204 & 595 & 1330 \\
\hline Refinement method & Full-matrix least-squares on $\left|F^{2}\right|$ & Full-matrix least-squares on $\left|F^{2}\right|$ & Full-matrix least-squares on $\left|F^{2}\right|$ \\
\hline$R_{\mathrm{int}}$ & 0.04 & 0.098 & 0.033 \\
\hline$R[I>2 \sigma(I)]$ & $R_{1}=0.05 ; \mathrm{w} R_{2}=0.13^{a}$ & $R_{1}=0.047 ; \mathrm{w} R_{2}=0.097^{a}$ & $R_{1}=0.055 ; \mathrm{w} R_{2}=0.12^{a}$ \\
\hline$R$ (all data) & $R_{1}=0.06 ; \mathrm{w} R_{2}=0.14$ & $R_{1}=0.089 ; \mathrm{w} R_{2}=0.112$ & $R_{1}=0.082 ; \mathrm{w} R_{2}=0.14$ \\
\hline Goodness of fit $(S)$ & 0.984 & 1.054 & 1.08 \\
\hline No. of variables & 145 & 92 & 163 \\
\hline $\begin{array}{l}\text { Largest difference map peak and } \\
\text { hole } \mathrm{e} \AA^{-3}\end{array}$ & 0.531 and -0.722 & 0.313 and -0.43 & 0.573 and -0.573 \\
\hline
\end{tabular}

${ }^{a} W=1 /\left[\sigma^{2}\left(F_{\mathrm{O}}\right)^{2}+(a P)^{2}+b P\right]$ where $P=\left[F_{\mathrm{O}}^{2}+2 F_{\mathrm{C}}^{2}\right] / 3 ; a=0.0936$ and $b=0.0$ for $\mathbf{I}, a=0.0499$ and $b=0.0$ for II, and $a=0.0478$ and $b=2.0188$ for III. 
TABLE 3

Atomic Coordinates $\left(\times 10^{4}\right)$ and Equivalent Isotropic Displacement Parameters $\left(\AA^{2} \times 10^{3}\right)$ for, I, $\left[\mathrm{C}_{3} \mathrm{~N}_{2} \mathrm{H}_{12}\right]\left[\mathrm{Al}\left(\mathrm{HPO}_{4}\right)\left(\mathrm{PO}_{4}\right)\right]$

\begin{tabular}{lrrrr}
\hline Atom & $x$ & $y$ & \multicolumn{1}{c}{$z$} & $U(\mathrm{eq})^{a}$ \\
\hline $\mathrm{P}(1)$ & $454(1)$ & $7519(1)$ & $9301(1)$ & $17(1)$ \\
$\mathrm{P}(2)$ & $2102(1)$ & $12403(1)$ & $16541(1)$ & $16(1)$ \\
$\mathrm{Al}(1)$ & $72(2)$ & $10103(2)$ & $12557(2)$ & $17(1)$ \\
$\mathrm{O}(1)$ & $1466(4)$ & $6581(4)$ & $8426(4)$ & $28(1)$ \\
$\mathrm{O}(2)$ & $1659(4)$ & $6350(4)$ & $9230(4)$ & $27(1)$ \\
$\mathrm{O}(3)$ & $935(4)$ & $8761(4)$ & $8515(4)$ & $22(1)$ \\
$\mathrm{O}(4)$ & $1008(4)$ & $8697(4)$ & $11314(4)$ & $23(1)$ \\
$\mathrm{O}(5)$ & $4010(4)$ & $13404(4)$ & $17593(4)$ & $26(1)$ \\
$\mathrm{O}(6)$ & $860(4)$ & $13534(4)$ & $16669(4)$ & $34(1)$ \\
$\mathrm{O}(7)$ & $1795(4)$ & $11385(4)$ & $14590(4)$ & $24(1)$ \\
$\mathrm{O}(8)$ & $1646(4)$ & $11043(4)$ & $17199(4)$ & $27(1)$ \\
$\mathrm{N}(1)$ & $5516(5)$ & $6971(5)$ & $9022(5)$ & $28(1)$ \\
$\mathrm{C}(1)$ & $4761(6)$ & $7947(6)$ & $8053(6)$ & $28(1)$ \\
$\mathrm{C}(2)$ & $4328(6)$ & $6965(6)$ & $6110(6)$ & $28(1)$ \\
$\mathrm{C}(3)$ & $3475(6)$ & $7908(6)$ & $5107(6)$ & $29(1)$ \\
$\mathrm{N}(2)$ & $3064(5)$ & $6981(5)$ & $3215(5)$ & $25(1)$ \\
\hline
\end{tabular}

${ }^{a} U$ (eq) is defined as one third of the trace of the orthogonalized $U_{i j}$ tensor.

(heating rate $10^{\circ} \mathrm{C} / \mathrm{min}$ ). I-III exhibit mass losses in region 200- $400^{\circ} \mathrm{C}$ (Fig. 1) and the mass loss corresponds with the loss of the amine molecule. Thus, the total mass losses of

TABLE 4

Selected Bond Distances and Angles in I, $\left[\mathrm{C}_{3} \mathrm{~N}_{2} \mathrm{H}_{12}\right]\left[\mathrm{Al}\left(\mathrm{HPO}_{4}\right)\left(\mathrm{PO}_{4}\right)\right]$

\begin{tabular}{|c|c|c|c|}
\hline Moiety & Distance $(\AA)$ & Moiety & Angle $\left(^{\circ}\right)$ \\
\hline $\mathrm{P}(1)-\mathrm{O}(1)$ & $1.491(3)$ & $\mathrm{O}(3)-\mathrm{P}(1)-\mathrm{O}(4)$ & $105.7(2)$ \\
\hline $\mathrm{P}(1)-\mathrm{O}(2)$ & $1.516(3)$ & $\mathrm{O}(5)-\mathrm{P}(2)-\mathrm{O}(6)$ & $113.8(2)$ \\
\hline $\mathrm{P}(1)-\mathrm{O}(3)$ & $1.549(3)$ & $\mathrm{O}(5)-\mathrm{P}(2)-\mathrm{O}(7)$ & $109.9(2)$ \\
\hline $\mathrm{P}(1)-\mathrm{O}(4)$ & $1.561(3)$ & $\mathrm{O}(6)-\mathrm{P}(2)-\mathrm{O}(7)$ & $108.7(2)$ \\
\hline $\mathrm{P}(2)-\mathrm{O}(5)$ & $1.500(3)$ & $\mathrm{O}(5)-\mathrm{P}(2)-\mathrm{O}(8)$ & 108.1(1) \\
\hline $\mathrm{P}(2)-\mathrm{O}(6)$ & $1.517(3)$ & $\mathrm{O}(6)-\mathrm{P}(2)-\mathrm{O}(8)$ & $109.3(2)$ \\
\hline $\mathrm{P}(2)-\mathrm{O}(7)$ & $1.533(3)$ & $\mathrm{O}(7)-\mathrm{P}(2)-\mathrm{O}(8)$ & $106.8(2)$ \\
\hline $\mathrm{P}(2)-\mathrm{O}(8)$ & $1.544(3)$ & $\mathrm{O}(3)^{1}-\mathrm{Al}(1)-\mathrm{O}(8)^{2}$ & $103.18(14)$ \\
\hline $\mathrm{Al}(1)-\mathrm{O}(3)^{1}$ & $1.734(3)$ & $\mathrm{O}(3)^{1}-\mathrm{Al}(1)-\mathrm{O}(7)$ & $115.2(2)$ \\
\hline $\mathrm{Al}(1)-\mathrm{O}(8)^{2}$ & $1.736(3)$ & $\mathrm{O}(8)^{2}-\mathrm{Al}(1)-\mathrm{O}(7)$ & $110.9(2)$ \\
\hline $\mathrm{A}(1)-\mathrm{O}(7)$ & $1.738(3)$ & $\mathrm{O}(3)^{1}-\mathrm{Al}(1)-\mathrm{O}(4)$ & $111.84(14)$ \\
\hline $\mathrm{Al}(1)-\mathrm{O}(4)$ & $1.752(3)$ & $\mathrm{O}(8)^{2}-\mathrm{Al}(1)-\mathrm{O}(4)$ & $111.5(2)$ \\
\hline Moiety & Angle $\left({ }^{\circ}\right)$ & $\mathrm{O}(7)-\mathrm{Al}(1)-\mathrm{O}(4)$ & $104.5(2)$ \\
\hline $\mathrm{O}(1)-\mathrm{P}(1)-\mathrm{O}(2)$ & $114.9(2)$ & $\begin{array}{l}\mathrm{P}(1)-\mathrm{O}(3)-\mathrm{Al}(1)^{1} \\
\mathrm{P}(1)-\mathrm{O}(4)-\mathrm{Al}(1)\end{array}$ & $\begin{array}{l}138.8(2) \\
134.2(2)\end{array}$ \\
\hline $\mathrm{O}(1)-\mathrm{P}(1)-\mathrm{O}(3)$ & $111.0(2)$ & $\mathrm{P}(2)-\mathrm{O}(7)-\mathrm{Al}(1)$ & $139.8(2)$ \\
\hline $\mathrm{O}(2)-\mathrm{P}(1)-\mathrm{O}(3)$ & $108.7(2)$ & $\mathrm{P}(2)-\mathrm{O}(8)-\mathrm{Al}(1)^{2}$ & $142.6(2)$ \\
\hline $\mathrm{O}(1)-\mathrm{P}(1)-\mathrm{O}(4)$ & $111.6(2)$ & & \\
\hline $\mathrm{O}(2)-\mathrm{P}(1)-\mathrm{O}(4)$ & $104.4(2)$ & & \\
\hline \multicolumn{4}{|c|}{ Organic moiety } \\
\hline Moiety & Distance (๕̊) & Moiety & Angle $\left(^{\circ}\right)$ \\
\hline $\mathrm{N}(1)-\mathrm{C}(1)$ & $1.484(6)$ & $\mathrm{N}(1)-\mathrm{C}(1)-\mathrm{C}(2)$ & 111.1(3) \\
\hline $\mathrm{C}(1)-\mathrm{C}(2)$ & $1.501(6)$ & $\mathrm{C}(1)-\mathrm{C}(2)-\mathrm{C}(3)$ & 111.3(4) \\
\hline $\mathrm{C}(2)-\mathrm{C}(3)$ & $1.508(6)$ & $\mathrm{N}(2)-\mathrm{C}(3)-\mathrm{C}(2)$ & 112.1(4) \\
\hline $\mathrm{C}(3)-\mathrm{N}(2)$ & $1.461(5)$ & & \\
\hline
\end{tabular}

Note. Symmetry transformations used to generate equivalent atoms: $1,-x,-y+2,-z+2 ; 2,-x,-y+2,-z+3$.
TABLE 5

Atomic Coordinates $\left(\times 10^{4}\right)$ and Equivalent Isotropic Displacement Parameters $\left(\AA^{2} \times 10^{3}\right)$ for, II, $\left[\mathrm{C}_{3} \mathrm{~N}_{2} \mathrm{H}_{12}\right]\left[\mathrm{NH}_{4}\right]\left[\mathrm{Al}\left(\mathrm{PO}_{4}\right)_{2}\right]$

\begin{tabular}{llccc}
\hline Atom & \multicolumn{1}{c}{$y$} & $y$ & $z(\mathrm{eq})$ \\
\hline $\mathrm{P}(1)$ & $6480(1)$ & $616(1)$ & $8692(1)$ & $20(1)$ \\
$\mathrm{Al}(1)$ & 7500 & 2500 & $6216(2)$ & $20(1)$ \\
$\mathrm{O}(1)$ & $5644(2)$ & $1206(4)$ & $8442(4)$ & $36(1)$ \\
$\mathrm{O}(2)$ & $6530(2)$ & $-1188(4)$ & $8865(4)$ & $35(1)$ \\
$\mathrm{O}(3)$ & $7012(2)$ & $1073(3)$ & $7314(4)$ & $35(1)$ \\
$\mathrm{O}(4)$ & $6823(2)$ & $1453(4)$ & $10122(4)$ & $41(1)$ \\
$\mathrm{N}(1)$ & $4902(2)$ & $2135(4)$ & $5756(4)$ & $28(1)$ \\
$\mathrm{C}(2)$ & $4633(3)$ & $548(5)$ & $5124(6)$ & $31(1)$ \\
$\mathrm{C}(1)$ & $4011(6)$ & $8(10)$ & $6332(11)$ & $49(4)$ \\
$\mathrm{N}(2)$ & 7500 & -2500 & $11256(10)$ & $34(2)$ \\
\hline
\end{tabular}

$26.1 \%$ for I, $26.2 \%$ for II, and $23.2 \%$ for III corresponds to the loss of the amine and -OH group for I (calcd. 28.9\%), amine and $\mathrm{NH}_{4}$ for II $30.2 \%$, and amine and dihydroxyhydrazine for III $28.4 \%$. The calcined sample is poorly crystalline by powder XRD and the majority of the lines correspond to the quartz analog of the aluminum phosphate, berlinite.

A suitable single crystal of each compound was carefully selected under a polarizing microscope and glued to a thin glass fiber with cyanoacrylate (super glue) adhesive. Singlecrystal structure determination by X-ray diffraction was

TABLE 6

Selected Bond Distances and Angles in II, $\left[\mathrm{C}_{3} \mathrm{~N}_{2} \mathrm{H}_{12}\right]\left[\mathrm{NH}_{4}\right]\left[\mathrm{Al}\left(\mathrm{PO}_{4}\right)_{2}\right]$

\begin{tabular}{lccc}
\hline Moiety & Distance $(\AA)$ & \multicolumn{1}{l}{ Moiety } & Angle $\left(^{\circ}\right)$ \\
\hline $\mathrm{P}(1)-\mathrm{O}(1)$ & $1.505(3)$ & $\mathrm{O}(1)-\mathrm{P}(1)-\mathrm{O}(4)$ & $108.8(2)$ \\
$\mathrm{P}(1)-\mathrm{O}(2)$ & $1.505(3)$ & $\mathrm{O}(2)-\mathrm{P}(1)-\mathrm{O}(3)$ & $106.8(2)$ \\
$\mathrm{P}(1)-\mathrm{O}(4)$ & $1.537(3)$ & $\mathrm{O}(1)-\mathrm{P}(1)-\mathrm{O}(3)$ & $110.5(2)$ \\
$\mathrm{P}(1)-\mathrm{O}(3)$ & $1.543(3)$ & $\mathrm{O}(4)-\mathrm{P}(1)-\mathrm{O}(3)$ & $107.4(2)$ \\
$\mathrm{Al}(1)-\mathrm{O}(4)^{1}$ & $1.719(3)$ & $\mathrm{O}(4)^{1}-\mathrm{Al}(1)-\mathrm{O}(4)^{2}$ & $112.8(2)$ \\
$\mathrm{Al}(1)-\mathrm{O}(4)^{2}$ & $1.719(3)$ & $\mathrm{O}(4)^{1}-\mathrm{Al}(1)-\mathrm{O}(3)^{3}$ & $106.0(2)$ \\
$\mathrm{A}(1)-\mathrm{O}(3)^{3}$ & $1.728(3)$ & $\mathrm{O}(4)^{2}-\mathrm{Al}(1)-\mathrm{O}(3)^{3}$ & $109.7(2)$ \\
$\mathrm{Al}(1)-\mathrm{O}(3)$ & $1.728(3)$ & $\mathrm{O}(4)^{1}-\mathrm{Al}(1)-\mathrm{O}(3)$ & $109.6(2)$ \\
& & $\mathrm{O}(4)^{2}-\mathrm{Al}(1)-\mathrm{O}(3)$ & $106.0(2)$ \\
$\mathrm{Moiety}$ & $\mathrm{Angle}\left({ }^{\circ}\right)$ & $\mathrm{O}(3)^{3}-\mathrm{Al}(1)-\mathrm{O}(3)$ & $112.9(2)$ \\
\hline $\mathrm{O}(2)-\mathrm{P}(1)-\mathrm{O}(1)$ & $112.9(2)$ & $\mathrm{P}(1)-\mathrm{O}(3)-\mathrm{Al}(1)$ & $150.8(2)$ \\
$\mathrm{O}(2)-\mathrm{P}(1)-\mathrm{O}(4)$ & $110.3(2)$ & & $157.6(2)$ \\
& \multicolumn{4}{c}{$\mathrm{Organic}(4)-\mathrm{Al}(1)^{4}$} & \\
$\mathrm{Moiety}$ & Distance $(\AA)$ & $\mathrm{Moiety}$ & Angle $\left(^{\circ}\right)$ \\
\hline $\mathrm{N}(1)-\mathrm{C}(2)$ & $1.496(5)$ & $\mathrm{N}(1)-\mathrm{C}(2)-\mathrm{C}(2)^{5}$ & $109.0(5)$ \\
$\mathrm{C}(2)-\mathrm{C}(2)^{5}$ & $1.547(9)$ & $\mathrm{N}(1)-\mathrm{C}(2)-\mathrm{C}(1)$ & $102.1(5)$ \\
$\mathrm{C}(2)-\mathrm{C}(1)$ & $1.550(10)$ & $\mathrm{C}(2)^{5}-\mathrm{C}(2)-\mathrm{C}(1)$ & $117.7(6)$ \\
\hline
\end{tabular}

Note. Symmetry transformations used to generate equivalent atoms: $1,-x,-y+\frac{1}{2}, z-\frac{1}{2} ; 2,-x+\frac{3}{2}, y, z-\frac{1}{2} ; 3,-x+\frac{3}{2},-y+\frac{1}{2}, z ; 4, x$, $-y+\frac{1}{2}, z+\frac{1}{2} ; 5,-x+1,-y,-z+1$. 
TABLE 7

Atomic Coordinates $\left(\times 10^{4}\right)$ and Equivalent Isotropic Displacement Parameters $\left(\AA^{2} \times 10^{3}\right)$ for, III, $\left[\mathrm{C}_{3} \mathbf{N}_{2} \mathbf{H}_{12}\right]$ $\left[\mathrm{OH}\left(\mathrm{NH}_{2}\right)_{2} \mathrm{OH}\right]_{0.5}\left[\mathrm{Al}\left(\mathrm{PO}_{4}\right)_{2}\right]$

\begin{tabular}{lrrrr}
\hline Atoms & \multicolumn{1}{c}{$x$} & \multicolumn{1}{c}{$z$} & $U$ \\
\hline $\mathrm{P}(1)$ & $2460(2)$ & $15827(2)$ & $7404(2)$ & $16(1)$ \\
$\mathrm{P}(2)$ & $1058(2)$ & $9767(2)$ & $3360(2)$ & $17(1)$ \\
$\mathrm{Al}(1)$ & $260(2)$ & $12542(2)$ & $4987(2)$ & $15(1)$ \\
$\mathrm{O}(1)$ & $3959(5)$ & $16378(5)$ & $7044(5)$ & $27(1)$ \\
$\mathrm{O}(2)$ & $3025(5)$ & $16190(4)$ & $9239(5)$ & $27(1)$ \\
$\mathrm{O}(3)$ & $959(5)$ & $16624(4)$ & $6574(5)$ & $26(1)$ \\
$\mathrm{O}(4)$ & $1699(5)$ & $14039(4)$ & $6710(5)$ & $26(1)$ \\
$\mathrm{O}(5)$ & $-742(5)$ & $9285(5)$ & $1848(5)$ & $32(1)$ \\
$\mathrm{O}(6)$ & $2613(5)$ & $9705(4)$ & $3032(5)$ & $26(1)$ \\
$\mathrm{O}(7)$ & $1446(5)$ & $11476(4)$ & $4322(5)$ & $20(1)$ \\
$\mathrm{O}(8)$ & $1147(5)$ & $8707(4)$ & $4567(5)$ & $23(1)$ \\
$\mathrm{N}(1)$ & $-4420(6)$ & $2345(5)$ & $-4691(6)$ & $25(1)$ \\
$\mathrm{C}(1)$ & $-4165(8)$ & $3712(7)$ & $-3413(8)$ & $26(2)$ \\
$\mathrm{C}(2)$ & $-2216(8)$ & $4594(7)$ & $-2443(8)$ & $30(2)$ \\
$\mathrm{C}(3)$ & $-1837(8)$ & $6259(7)$ & $-1511(8)$ & $32(2)$ \\
$\mathrm{N}(2)$ & $-2706(6)$ & $6396(5)$ & $-438(6)$ & $26(1)$ \\
$\mathrm{N}(10)$ & $4844(9)$ & $9974(8)$ & $751(8)$ & $58(2)$ \\
$\mathrm{O}(10)$ & $2933(6)$ & $9200(5)$ & $233(6)$ & $47(1)$ \\
\hline & & & &
\end{tabular}

TABLE 8

Selected Bond Distances and Bond Angles in III, $\left[\mathrm{C}_{3} \mathrm{~N}_{2} \mathrm{H}_{12}\right]\left[\mathrm{OH}\left(\mathrm{NH}_{2}\right)_{2} \mathrm{OH}\right]_{0.5}\left[\mathrm{Al}\left(\mathrm{PO}_{4}\right)_{2}\right]$

\begin{tabular}{|c|c|c|c|}
\hline Moiety & Distance & Moiety & Angle $\left({ }^{\circ}\right)$ \\
\hline $\mathrm{P}(1)-\mathrm{O}(1)$ & $1.502(4)$ & $\mathrm{O}(2)-\mathrm{P}(1)-\mathrm{O}(4)$ & $107.9(2)$ \\
\hline $\mathrm{P}(1)-\mathrm{O}(2)$ & $1.514(4)$ & $\mathrm{O}(3)-\mathrm{P}(1)-\mathrm{O}(4)$ & $108.5(2)$ \\
\hline $\mathrm{P}(1)-\mathrm{O}(3)$ & $1.544(4)$ & $\mathrm{O}(5)-\mathrm{P}(2)-\mathrm{O}(6)$ & $115.5(3)$ \\
\hline $\mathrm{P}(1)-\mathrm{O}(4)$ & $1.544(4)$ & $\mathrm{O}(5)-\mathrm{P}(2)-\mathrm{O}(8)$ & $110.8(2)$ \\
\hline $\mathrm{P}(2)-\mathrm{O}(5)$ & $1.505(4)$ & $\mathrm{O}(6)-\mathrm{P}(2)-\mathrm{O}(8)$ & $106.1(2)$ \\
\hline $\mathrm{P}(2)-\mathrm{O}(6)$ & $1.514(4)$ & $\mathrm{O}(5)-\mathrm{P}(2)-\mathrm{O}(7)$ & $110.7(2)$ \\
\hline $\mathrm{P}(2)-\mathrm{O}(8)$ & $1.552(4)$ & $\mathrm{O}(6)-\mathrm{P}(2)-\mathrm{O}(7)$ & $106.1(2)$ \\
\hline $\mathrm{P}(2)-\mathrm{O}(7)$ & $1.555(4)$ & $\mathrm{O}(8)-\mathrm{P}(2)-\mathrm{O}(7)$ & 107.1(2) \\
\hline $\mathrm{Al}(1)-\mathrm{O}(4)$ & $1.724(4)$ & $\mathrm{O}(4)-\mathrm{Al}(1)-\mathrm{O}(3)^{1}$ & $107.7(2)$ \\
\hline $\mathrm{Al}(1)-\mathrm{O}(3)^{1}$ & $1.732(4)$ & $\mathrm{O}(4)-\mathrm{Al}(1)-\mathrm{O}(8)^{2}$ & $110.4(2)$ \\
\hline $\mathrm{Al}(1)-\mathrm{O}(8)^{2}$ & $1.739(4)$ & $\mathrm{O}(3)^{1}-\mathrm{Al}(1)-\mathrm{O}(8)^{2}$ & $110.3(2)$ \\
\hline $\mathrm{Al}(1)-\mathrm{O}(7)$ & $1.750(4)$ & $\mathrm{O}(4)-\mathrm{Al}(1)-\mathrm{O}(7)$ & $110.5(2)$ \\
\hline Moiety & Angle $\left(^{\circ}\right)$ & $\begin{array}{l}\mathrm{O}(3)^{1}-\mathrm{Al}(1)-\mathrm{O}(7) \\
\mathrm{O}(8)-\mathrm{Al}(1)-\mathrm{O}(7)\end{array}$ & $\begin{array}{l}108.7(2) \\
109.3(2)\end{array}$ \\
\hline $\mathrm{O}(1)-\mathrm{P}(1)-\mathrm{O}(2)$ & $112.9(2)$ & $\begin{array}{l}\mathrm{P}(1)-\mathrm{O}(3)-\mathrm{Al}(1)^{1} \\
\mathrm{P}(1)-\mathrm{O}(4)-\mathrm{Al}(1)\end{array}$ & $\begin{array}{l}151.8(3) \\
1458(3)\end{array}$ \\
\hline $\mathrm{O}(1)-\mathrm{P}(1)-\mathrm{O}(3)$ & $110.0(2)$ & $\begin{array}{l}P(1)-U(4)-A(1) \\
P(2)-O(7)-A l(1)\end{array}$ & $\begin{array}{l}145.8(3) \\
135.0(2)\end{array}$ \\
\hline $\mathrm{O}(2)-\mathrm{P}(1)-\mathrm{O}(3)$ & $107.5(2)$ & $\mathrm{P}(2)-\mathrm{O}(8)-\mathrm{Al}(1)^{2}$ & $140.0(3)$ \\
\hline $\mathrm{O}(1)-\mathrm{P}(1)-\mathrm{O}(4)$ & $109.9(2)$ & & $140.0(J)$ \\
\hline \multicolumn{4}{|c|}{ Organic moiety } \\
\hline Moiety & Distance & Moiety & Angle $\left({ }^{\circ}\right)$ \\
\hline $\mathrm{N}(1)-\mathrm{C}(1)$ & $1.487(7)$ & $\mathrm{N}(1)-\mathrm{C}(1)-\mathrm{C}(2)$ & $109.9(5)$ \\
\hline$C(1)-C(2)$ & $1.517(8)$ & $C(3)-C(2)-C(1)$ & $115.3(5)$ \\
\hline $\mathrm{C}(2)-\mathrm{C}(3)$ & $1.513(8)$ & $\mathrm{N}(2)-\mathrm{C}(3)-\mathrm{C}(2)$ & $113.2(5)$ \\
\hline $\mathrm{C}(3)-\mathrm{N}(2)$ & $1.492(8)$ & $\mathrm{O}(10)-\mathrm{N}(10)-\mathrm{N}(10)^{3}$ & $111.7(7)$ \\
\hline $\mathrm{N}(10)-\mathrm{O}(10)$ & $1.509(8)$ & & \\
\hline $\mathrm{N}(10)-\mathrm{N}(10)^{3}$ & $1.535(12)$ & & \\
\hline
\end{tabular}

Note. Symmetry transformations used to generate equivalent atoms: 1 , $-x,-y+3,-z+1 ; 2,-x,-y+2,-z+1 ; 3,-x+1,-y+2,-z$.

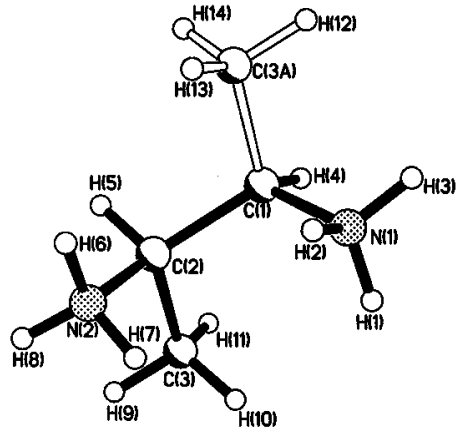

(a)

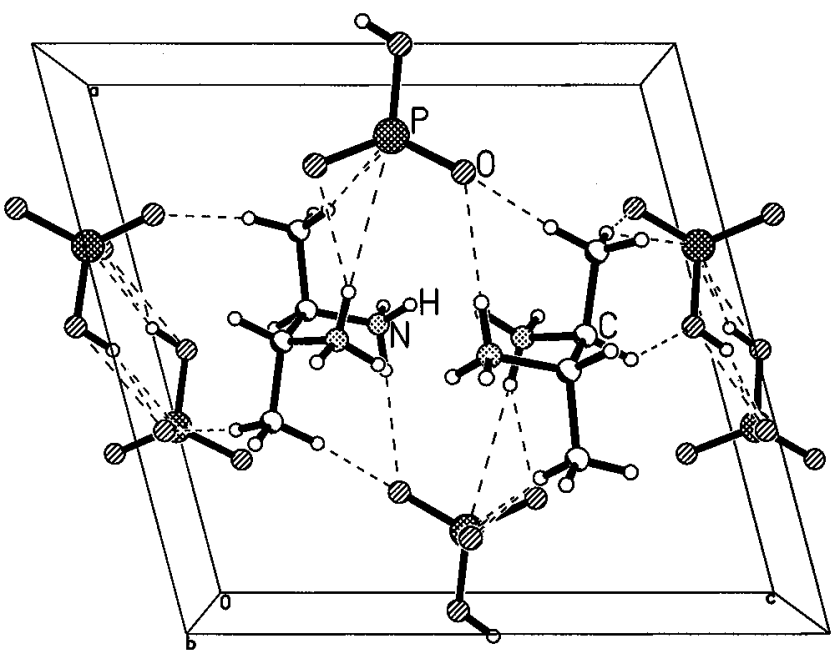

(b)

FIG. 2. (a) ORTEP plot of the 1,2-DAP-P. Thermal ellipsoids are given at $50 \%$ probability. (b) Packing diagram of 1,2-DAP-P showing the layer-like structure formed by hydrogen bond and interactions along with the position of the amine.

performed on a Siemens Smart-CCD diffractometer equipped with a normal focus, $2.4 \mathrm{~kW}$ sealed tube X-ray source (MoK $\alpha$ radiation, $\lambda=0.71073 \AA$ ) operating at $50 \mathrm{kV}$ and $40 \mathrm{~mA}$. A hemisphere of intensity data were collected using SMART software (14) at room temperature in 1321 frames with $\omega$ scans (width of $0.30^{\circ}$ and exposure time of $20 \mathrm{~s}$ per frame) in the $2 \theta$ range 3 to $46.5^{\circ}$. The total collected data were reduced using SAINT (14) program and the orientation matrix along with the detector and the cell parameters were refined for every 40 frames on all the measured reflections. Pertinent experimental details for the structure determinations are presented in Table 1. 

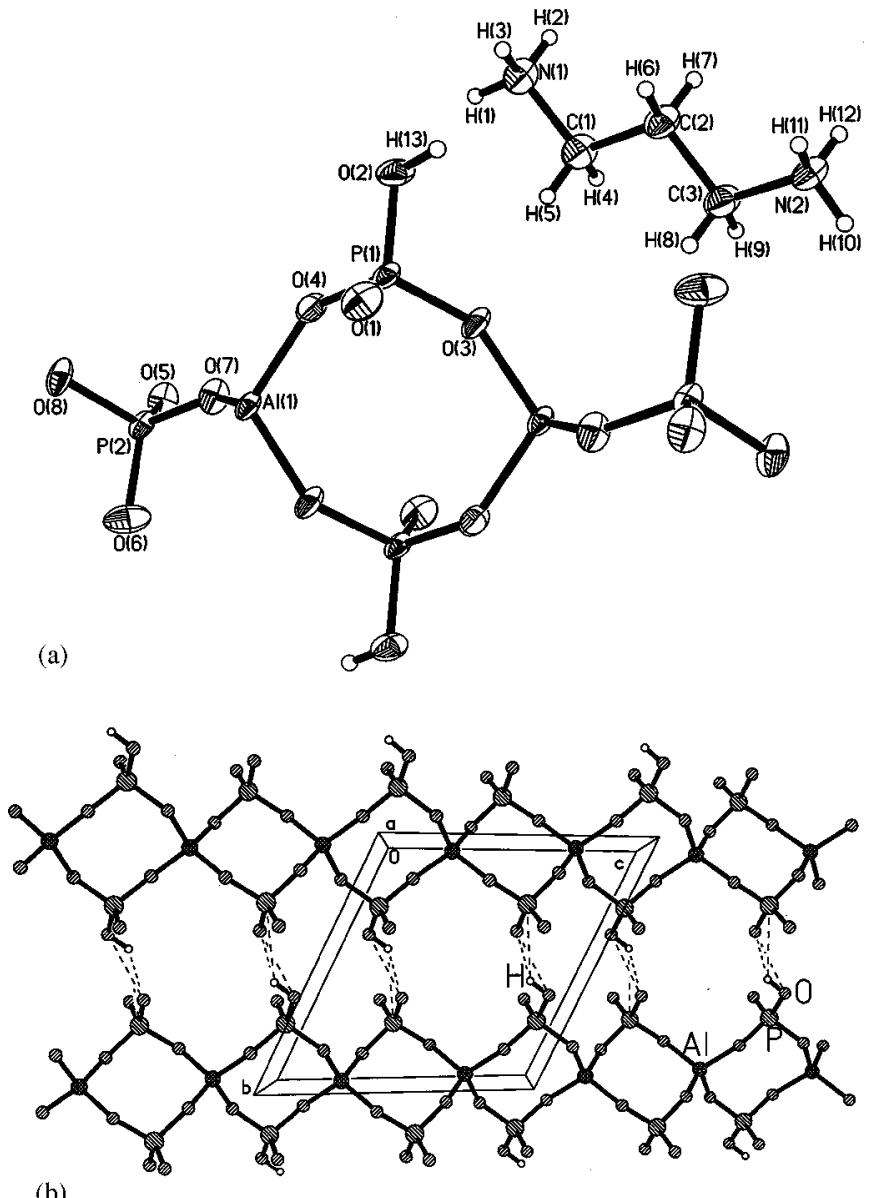

(b)

FIG. 3. (a) ORTEP plot of $\left[\mathrm{C}_{3} \mathrm{~N}_{2} \mathrm{H}_{12}\right]\left[\mathrm{Al}\left(\mathrm{HPO}_{4}\right)\left(\mathrm{PO}_{4}\right)\right]$, I. Asymmetric unit is labeled. Thermal ellipsoids are given at $50 \%$ probability. (b) Structure of I along the $a$ axis showing the chains. Note that the chains interact via hydrogen bonded interactions (dotted lines). Amine molecules are not shown for clarity.

An empirical absorption correction based on symmetry equivalent reflections was applied using the SADABS (15) program. Other effects, such as absorption by glass fiber etc. were simultaneously corrected. The structures of I, II, and III were solved by direct methods using SHELXS-86 (16), which readily established all the heavy atom positions (Al and $\mathrm{P}$ ) and facilitated the identification of most of the other fragments $(\mathrm{O}, \mathrm{C}, \mathrm{N}$, and $\mathrm{H})$ from difference Fourier maps. The amine molecule, 1,2-DAP, in II is found to be disordered. All the hydrogen positions were initially located in the difference Fourier maps, and for the final refinement, the hydrogen atoms were placed geometrically and held in the riding mode. The last cycles of refinement included atomic positions for all the atoms, anisotropic thermal parameters for all the nonhydrogen atoms and isotropic thermal parameters for all the hydrogen atoms. Full-matrix-least-squares structure refinement against $\left|F^{2}\right|$ was carried out using SHELXTL-PLUS (17) package of programs. Details of the
TABLE 9

Selected Hydrogen Bond Interactions in Compounds I-III

\begin{tabular}{|c|c|c|c|}
\hline Moiety & Distance $(\AA)$ & Moiety & Angle $\left({ }^{\circ}\right)$ \\
\hline \multicolumn{4}{|c|}{ I } \\
\hline $\mathrm{O}(1)-\mathrm{H}(2)$ & $1.866(6)$ & $\mathrm{O}(1)-\mathrm{H}(2)-\mathrm{N}(1)$ & $174.7(5)$ \\
\hline $\mathrm{O}(5)-\mathrm{H}(3)$ & $1.952(6)$ & $\mathrm{O}(5)-\mathrm{H}(3)-\mathrm{N}(1)$ & $153.3(5)$ \\
\hline $\mathrm{O}(4)-\mathrm{H}(10)$ & $2.079(6)$ & $\mathrm{O}(4)-\mathrm{H}(10)-\mathrm{N}(2)$ & $170.6(5)$ \\
\hline $\mathrm{O}(1)-\mathrm{H}(11)$ & $1.929(6)$ & $\mathrm{O}(1)-\mathrm{H}(11)-\mathrm{N}(2)$ & $157.7(5)$ \\
\hline $\mathrm{O}(5)-\mathrm{H}(12)$ & $1.877(6)$ & $\mathrm{O}(5)-\mathrm{H}(12)-\mathrm{N}(2)$ & $173.5(5)$ \\
\hline $\mathrm{O}(6)-\mathrm{H}(13)$ & $1.749(5)$ & $\mathrm{O}(6)-\mathrm{H}(13)-\mathrm{O}(2)$ & $142.4(5)$ \\
\hline $\mathrm{O}(3)-\mathrm{H}(5)$ & $2.491(6)$ & $\mathrm{O}(3)-\mathrm{H}(5)-\mathrm{C}(1)$ & $174.4(5)$ \\
\hline \multicolumn{4}{|c|}{ II } \\
\hline $\mathrm{O}(1)-\mathrm{H}(6)$ & $1.879(5)$ & $\mathrm{O}(1)-\mathrm{H}(6)-\mathrm{N}(1)$ & $168.5(4)$ \\
\hline $\mathrm{O}(1)-\mathrm{H}(7)$ & $1.876(5)$ & $\mathrm{O}(1)-\mathrm{H}(7)-\mathrm{N}(1)$ & $162.7(4)$ \\
\hline $\mathrm{O}(2)-\mathrm{H}(8)$ & $1.922(5)$ & $\mathrm{O}(2)-\mathrm{H}(8)-\mathrm{N}(1)$ & $169.9(4)$ \\
\hline $\mathrm{O}(2)-\mathrm{H}(11)$ & $2.186(2)$ & $\mathrm{O}(2)-\mathrm{H}(11)-\mathrm{N}(2)$ & $157(5)$ \\
\hline $\mathrm{O}(3)-\mathrm{H}(11)$ & $2.55(5)$ & $\mathrm{O}(3)-\mathrm{H}(11)-\mathrm{N}(2)$ & $142(4)$ \\
\hline $\mathrm{O}(2)-\mathrm{H}(12)$ & $1.86(7)$ & $\mathrm{O}(2)-\mathrm{H}(12)-\mathrm{N}(2)$ & $159(6)$ \\
\hline $\mathrm{O}(4)-\mathrm{H}(1)$ & $2.550(9)$ & $\mathrm{O}(4)-\mathrm{H}(1)-\mathrm{C}(1)$ & $170.8(10)$ \\
\hline \multicolumn{4}{|c|}{ III } \\
\hline $\mathrm{O}(6)-\mathrm{H}(1)$ & $2.134(6)$ & $\mathrm{O}(6)-\mathrm{H}(1)-\mathrm{N}(1)$ & $143.7(6)$ \\
\hline $\mathrm{O}(7)-\mathrm{H}(1)$ & $2.401(8)$ & $\mathrm{O}(7)-\mathrm{H}(1)-\mathrm{N}(1)$ & $150.3(6)$ \\
\hline $\mathrm{O}(6)-\mathrm{H}(2)$ & $1.914(6)$ & $\mathrm{O}(6)-\mathrm{H}(2)-\mathrm{N}(1)$ & $173.0(6)$ \\
\hline $\mathrm{O}(1)-\mathrm{H}(3)$ & $1.889(7)$ & $\mathrm{O}(1)-\mathrm{H}(3)-\mathrm{N}(1)$ & $161.3(6)$ \\
\hline $\mathrm{O}(5)-\mathrm{H}(10)$ & $1.990(6)$ & $\mathrm{O}(5)-\mathrm{H}(10)-\mathrm{N}(2)$ & $150.8(6)$ \\
\hline $\mathrm{O}(2)-\mathrm{H}(11)$ & $1.977(6)$ & $\mathrm{O}(2)-\mathrm{H}(11)-\mathrm{N}(2)$ & 141.3(3) \\
\hline $\mathrm{O}(1)-\mathrm{H}(12)$ & $2.036(7)$ & $\mathrm{O}(1)-\mathrm{H}(12)-\mathrm{N}(2)$ & $143.6(5)$ \\
\hline $\mathrm{O}(1)-\mathrm{H}(14)$ & $2.414(8)$ & $\mathrm{O}(1)-\mathrm{H}(14)-\mathrm{N}(10)$ & 177.2(9) \\
\hline $\mathrm{O}(2)-\mathrm{H}(15)$ & $1.950(6)$ & $\mathrm{O}(2)-\mathrm{H}(15)-\mathrm{O}(10)$ & $162.3(7)$ \\
\hline
\end{tabular}

final refinements are given in Table 2. The final atomic coordinates, bond distances, and angles are presented in Tables 3 and 4 for $\mathbf{I}$, in Tables 5 and 6 for II, and in Tables 7 and 8 for III.

\section{RESULTS AND DISCUSSION}

The three one-dimensional AlPOs, I-III, are formed by the reaction of the $\mathrm{Al}^{3+}$ ions with amine phosphates, viz. 1,3-DAP-P and 1,2-DAP-P. Since 1,2-DAP-P was synthesized for the first time, its structure was determined. The structure of 1,2-DAP-P is shown in Fig. 2. As can be seen, the di-hydrogen phosphate units interact with each other via noncovalent interactions, forming a layer-like arrangement with apertures. The amine molecule is disordered and sits in the middle of the aperture (Fig. 2b) and forms hydrogen bonds with the phosphate moieties. Similar amine phosphate structures have been described recently (18).

The asymmetric unit of I contains 16 nonhydrogen atoms as shown in Fig. 3a. The structure is of a polymeric onedimensional aluminum phosphate of the formula $\left[\mathrm{Al}\left(\mathrm{HPO}_{4}\right)\left(\mathrm{PO}_{4}\right)\right]^{2-}$, as can be seen from Fig. 3b. Charge compensation in this compound is achieved by the presence of diprotonated 1,3-diaminopropane (1,3-DAP). The Al 

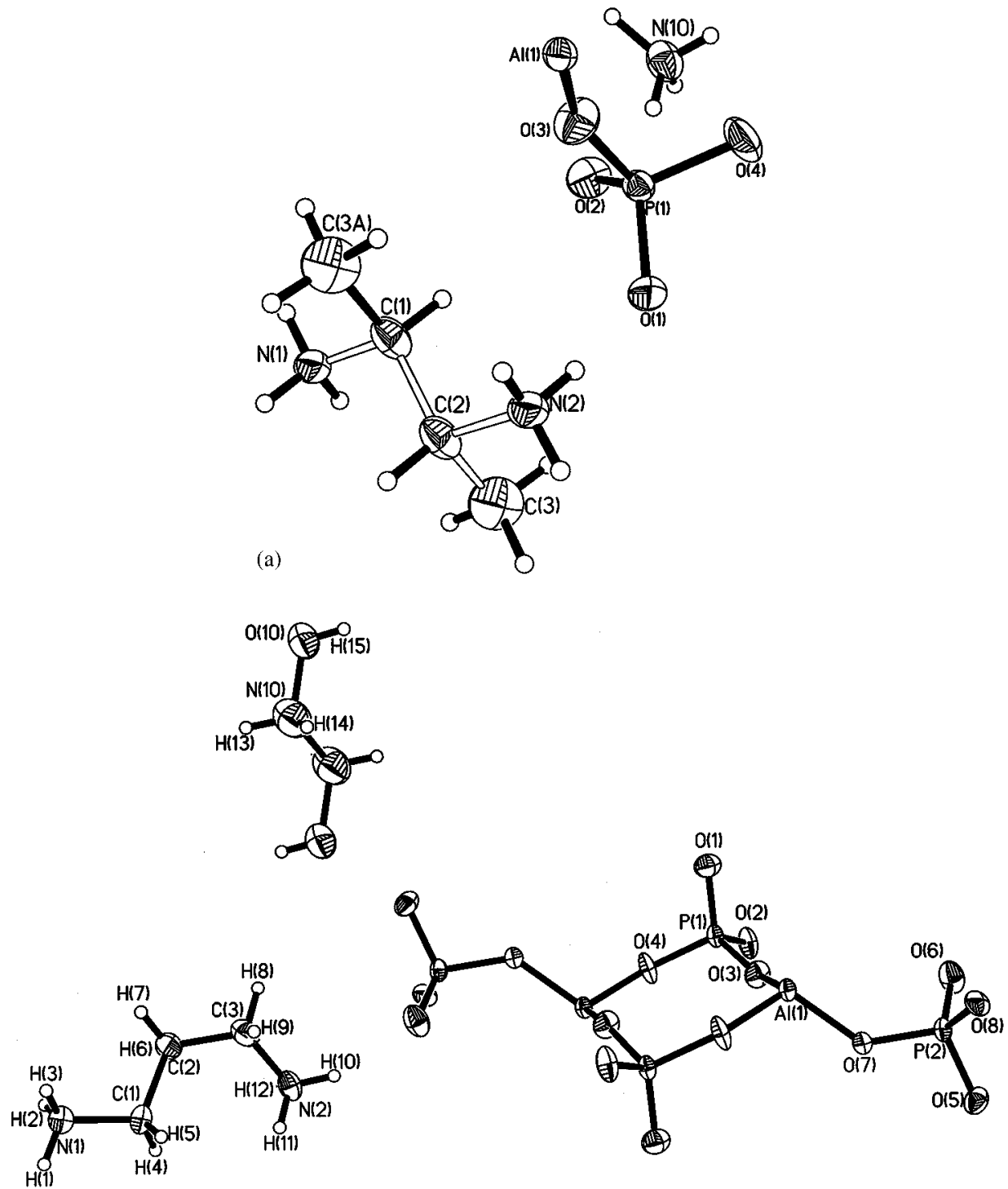

(a)

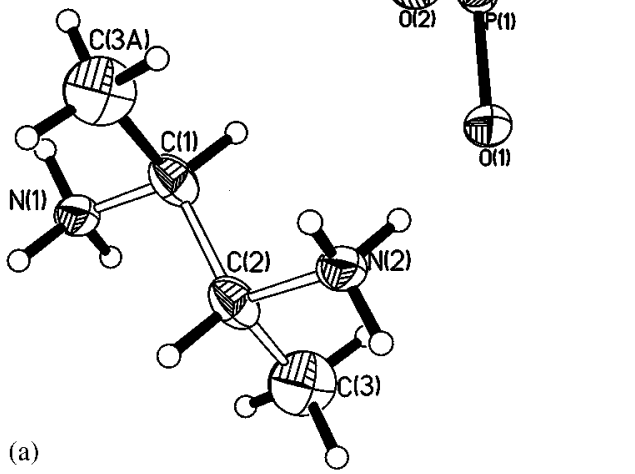

(b)

FIG. 4. (a) ORTEP plot of $\left[\mathrm{H}_{3} \mathrm{NCH}_{2} \mathrm{CH}\left(\mathrm{NH}_{3}\right) \mathrm{CH}_{3}\right]\left[\mathrm{NH}_{4}\right]\left[\mathrm{Al}\left(\mathrm{PO}_{4}\right)_{2}\right]$, II. Thermal ellipsoids are given at $50 \%$ probability. Note the disorder in the amine. (b) ORTEP plot of $\left[\mathrm{H}_{3} \mathrm{~N}\left(\mathrm{CH}_{2}\right)_{3} \mathrm{NH}_{3}\right]\left[\mathrm{N}_{2} \mathrm{H}_{6} \mathrm{O}_{2}\right]_{0.5}\left[\mathrm{Al}\left(\mathrm{PO}_{4}\right)_{2}\right]$, III. Asymmetric unit is labeled. Thermal ellipsoids are given at 50\% probability.

atoms are tetrahedrally coordinated with average Al-O distances of $1.739 \AA$ and O-Al-O angle of $109.5^{\circ}$. These are typical values of $\mathrm{Al}$ in tetrahedral coordination. The structure contains two crystallographically distinct $\mathrm{P}$ atoms with $\mathrm{P}-\mathrm{O}$ distances in the range 1.492(3)-1.562(3) $\AA$ $\left([\mathrm{P}(1)-\mathrm{O}]_{\mathrm{av} .}=1.531\right.$ and $\left.[\mathrm{P}(2)-\mathrm{O}]_{\mathrm{av} .}=1.524 \AA\right)$. Both the $\mathrm{P}$ atoms have the same $\mathrm{O}-\mathrm{P}-\mathrm{O}$ bond angle of $109.4^{\circ}$. The various geometric parameters are in agreement with that observed earlier for similar compounds $(1-8)$. Bond valence sum calculations (19) indicate that $\mathrm{O}(2)$ is protonated in agreement with the proton position observed in difference Fourier map.
The $\mathrm{AlO}_{4}$ and $\mathrm{PO}_{4}$ tetrahedra in $\mathbf{I}$ alternate forming four-membered rings, which get linked through their corners to form one-dimensional chains. The arrangement of the individual tetrahedra are such that every other fourmembered ring in the chain is formed by either a $\mathrm{PO}_{4}$ or a $\mathrm{HPO}_{4}$ unit as shown in Fig. 3b. The -OH group of the $\mathrm{HPO}_{4}$ interacts with the $\mathrm{PO}_{4}$ group of the neighbouring chain forming pseudoapertures (Fig. 3b). The individual chain units are held together by hydrogen bond interactions involving the amine. It is to be noted that the inorganic chain, $\left[\mathrm{Al}\left(\mathrm{PO}_{4}\right)\left(\mathrm{HPO}_{4}\right)\right]$, has been observed for the first time in AlPOs, though such chain compositions have been 


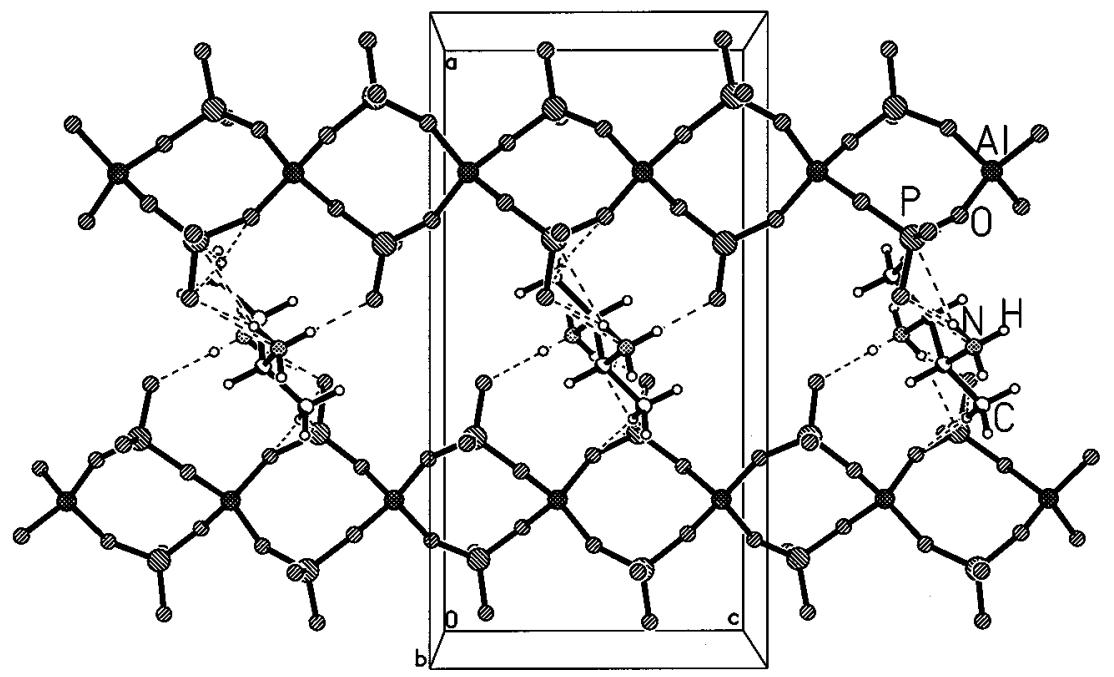

FIG. 5. Structure of II along the $b$ axis showing the chain and the amine molecules. Dotted lines represent the hydrogen bond interactions.

observed in edge-shared AlPOs (6b) and gallium phosphates (12). The various hydrogen bond interactions in $\mathbf{I}$ are listed in Table 9.

The asymmetric units of II and III contain 10 and 18 nonhydrogen atoms respectively as shown in Figs. 4a and $4 \mathrm{~b}$. The structures of these two AlPOs consist of polymeric one-dimensional aluminum phosphate anions with the formula $\left[\mathrm{Al}\left(\mathrm{PO}_{4}\right)_{2}\right]^{3-}$. Unlike in $\mathbf{I}$, the charge compensation in II and III is achieved by the presence of two different cations. While $\left[\mathrm{NH}_{4}\right]^{+}$and diprotonated 1,2-DAP cations compensate the framework negative charge in II, $0.5\left[\mathrm{OH}\left(\mathrm{NH}_{2}\right)_{2} \mathrm{OH}\right]^{2+}$ and diprotonated 1,3-DAP satisfies the requirement in III. It is likely that the $\left[\mathrm{NH}_{4}\right]^{+}$and the $0.5\left[\mathrm{OH}\left(\mathrm{NH}_{2}\right)_{2} \mathrm{OH}\right]^{2+}$ have resulted from the transformation of the amine. The dihydroxyhydrazine molecule formed in III has been observed for the first time in such structures. Formation of newer types of amines under hydrothermal conditions itself is known (20).

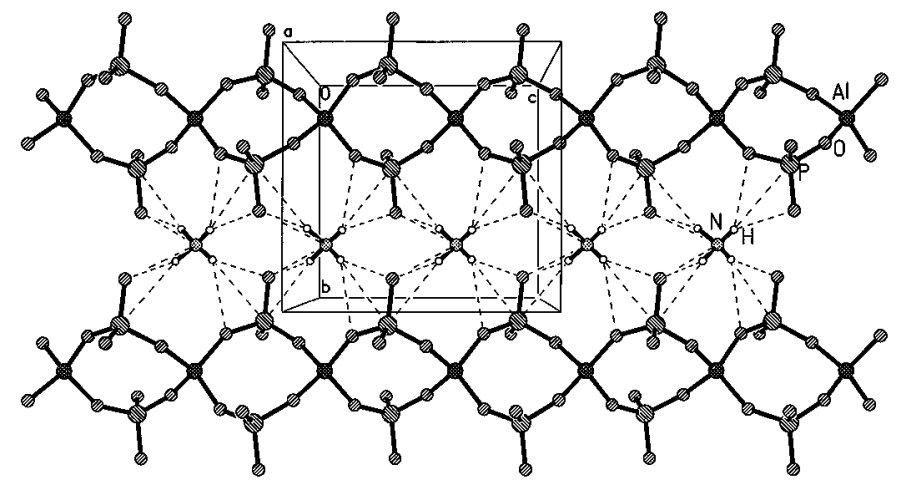

FIG. 6. Structure of II along the $a$ axis showing the chain and the $\left[\mathrm{NH}_{4}\right]^{+}$cation. Note the $\left[\mathrm{NH}_{4}\right]^{+}$cations occupy spaces in between the chains. Dotted lines represent the hydrogen bond interactions.
In II, the aluminum atoms occupy a special position and are tetrahedrally coordinated by four $\mathrm{O}$ atoms with $\mathrm{Al}-\mathrm{O}$ contacts varying between $1.719(3)-1.729(3) \AA$ and O-Al-O angles in the range 106.0(2)-112.9(2) . In III, the average $\mathrm{Al}-\mathrm{O}$ distance of $1.736 \AA$ and the $\mathrm{O}-\mathrm{Al}-\mathrm{O}$ angle of $109.5^{\circ}$ also result from the tetrahedral coordination by oxygen atoms. In both II and III, the $\mathrm{Al}$ atoms are connected to $\mathrm{P}$ atoms via $\mathrm{Al}-\mathrm{O}-\mathrm{P}$ linkages. In II, the independent $\mathrm{P}$ atom is tetrahedrally coordinated to four $\mathrm{O}$ atoms. Two of these oxygen atoms are bonded to $\mathrm{Al}$ atoms with $\mathrm{P}-\mathrm{O}$ bond lengths of $1.537(3)$ and $1.543(3) \AA$. The remaining $\mathrm{P}-\mathrm{O}$ bonds (i.e., $\mathrm{P}(1)-\mathrm{O}(1)$ and $\mathrm{P}(1)-\mathrm{O}(4)$ of lengths $1.505 \AA$ each) both clearly have multiple bond characters, and are comparable with those in, for example, $\mathrm{H}_{3} \mathrm{PO}_{4} \cdot 0.5 \mathrm{H}_{2} \mathrm{O}(1.485$ and $1.495 \AA$, respectively) (21). In III, the two distinct $\mathrm{P}$ atoms also show similar bonding with average $\mathrm{P}-\mathrm{O}$ bond distances of $1.529 \AA$ and O-P-O angle of $109.5^{\circ}$. These values agree well with those reported earlier for similar compounds (1-8).

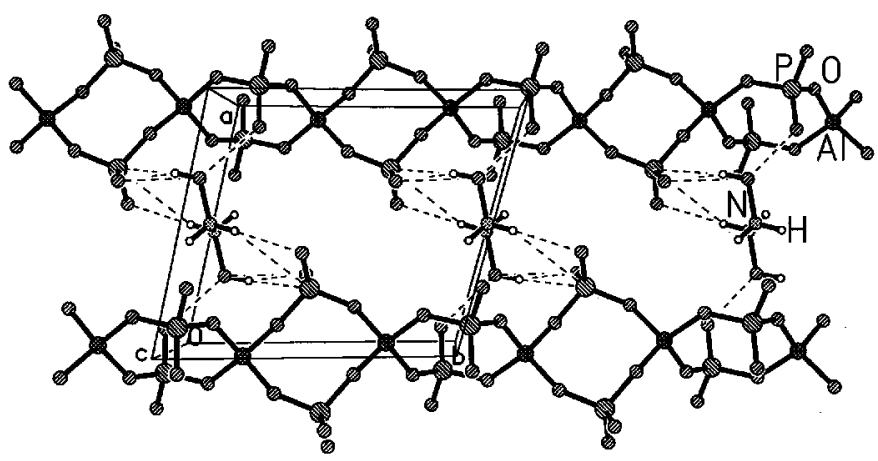

FIG. 7. Structure of III along the $c$ axis showing the chain and the dihydroxyhydrazine molecule. Dotted lines represent the hydrogen bond interactions. 


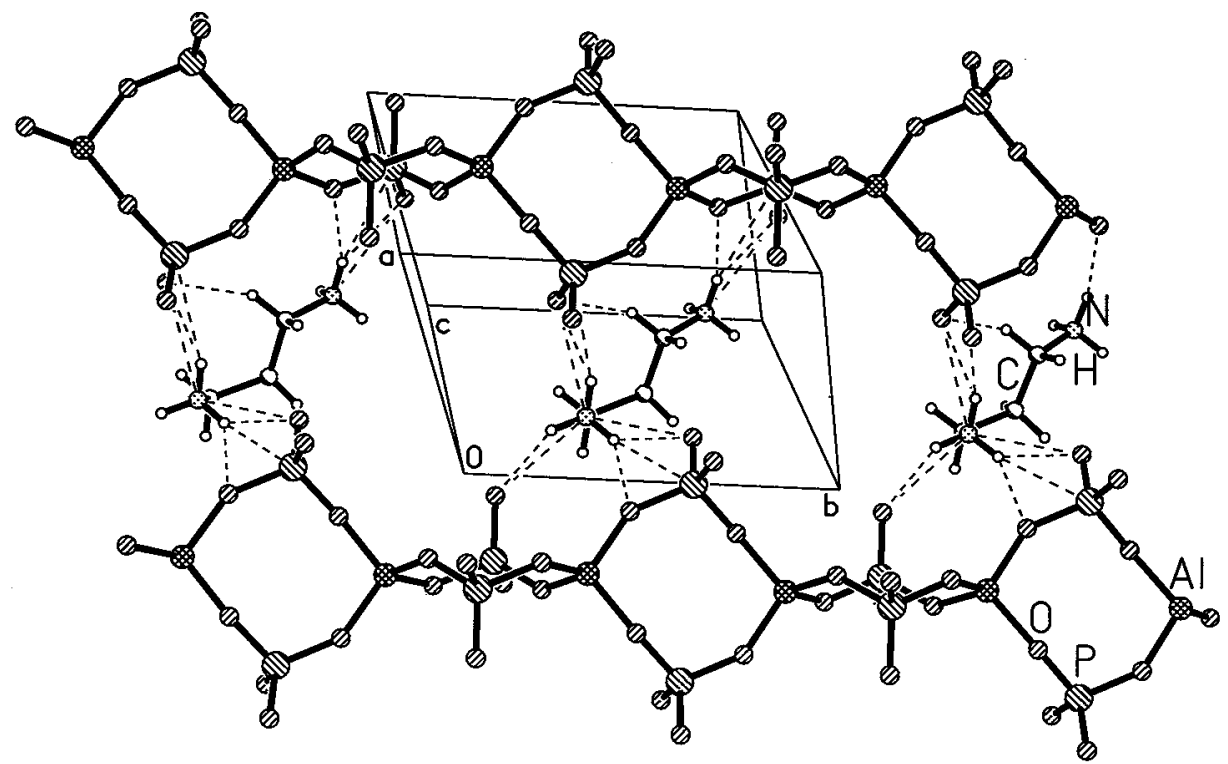

FIG. 8. Structure of III showing the inorganic chains and the 1,3-DAP molecule. Note the strong hydrogen bond interactions between the chain and the amine molecule.

The structures of II and III are formed by strictly alternating $\mathrm{AlO}_{4}$ and $\mathrm{PO}_{4}$ tetrahedra linked through their vertices. In II, the tetrahedra are connected to form fourmembered rings, which are corner-linked, forming one-dimensional chains (Fig. 5). The charge balancing 1,2-DAP and $\mathrm{NH}_{4}$ cations are situated in between the chains (Figs. 5 and 6). In Figs. 7 and 8, we show the chains along with the cations present in III. In both II and III, the charge balancing cations coexist in between the inorganic chains and interact with the framework via hydrogen bonds. The hydrogen bond interactions present in II and III are listed in Table 9.

\section{CONCLUSIONS}

Three aluminum phosphates, $\left[\mathrm{H}_{3} \mathrm{~N}\left(\mathrm{CH}_{2}\right)_{3} \mathrm{NH}_{3}\right][\mathrm{Al}$ $\left.\left(\mathrm{HPO}_{4}\right)\left(\mathrm{PO}_{4}\right)\right], \mathbf{I},\left[\mathrm{H}_{3} \mathrm{NCH}_{2} \mathrm{CH}\left(\mathrm{NH}_{3}\right) \mathrm{CH}_{3}\right]\left[\mathrm{NH}_{4}\right][\mathrm{Al}$ $\left.\left(\mathrm{PO}_{4}\right)_{2}\right]$, II, $\left[\mathrm{H}_{3} \mathrm{~N}\left(\mathrm{CH}_{2}\right)_{3} \mathrm{NH}_{3}\right]\left[\mathrm{N}_{2} \mathrm{H}_{6} \mathrm{O}_{2}\right]_{0.5}\left[\mathrm{Al}\left(\mathrm{PO}_{4}\right)_{2}\right]$,

TABLE 10

List of the Known AIPOs with One-Dimensional Structure

\begin{tabular}{|c|c|c|c|c|c|c|c|c|c|}
\hline \multirow[b]{2}{*}{ Compound } & \multicolumn{7}{|c|}{ Lattice parameters } & \multirow[b]{2}{*}{ Structure type } & \multirow[b]{2}{*}{ Ref. } \\
\hline & $a(\AA)$ & $b(\AA)$ & $c(\AA)$ & $\alpha\left({ }^{\circ}\right)$ & $\beta\left({ }^{\circ}\right)$ & $\gamma\left({ }^{\circ}\right)$ & $\begin{array}{l}\text { Space } \\
\text { group }\end{array}$ & & \\
\hline $\mathrm{Et}_{3} \mathrm{NH}\left[\mathrm{Al}\left(\mathrm{PO}_{4}\right)_{2}\right]$ & $12.073(2)$ & $13.201(2)$ & $8.522(1)$ & 90.0 & $97.2(1)$ & 90.0 & $P 2_{1} / n$ & Corner shared & $10 \mathrm{~b}$ \\
\hline$\left[\mathrm{C}_{2} \mathrm{~N}_{2} \mathrm{H}_{10}\right]\left[\mathrm{Al}\left(\mathrm{PO}_{4}\right)_{2}\right] \mathrm{H}_{2} \mathrm{O}$ & $8.052(6)$ & $8.760(2)$ & $17.037(7)$ & 90.0 & 90.0 & 90.0 & $P b n b$ & Corner shared & $9 \mathrm{a}$ \\
\hline $\mathrm{Na}_{4}\left[\mathrm{Al}\left(\mathrm{PO}_{4}\right)_{22}(\mathrm{OH})\right]$ & $15.279(3)$ & $14.660(3)$ & $6.947(3)$ & 90.0 & 90.0 & 90.0 & $\mathrm{Pbcm}$ & Tancoite type & $11 \mathrm{~b}$ \\
\hline$\left[\mathrm{C}_{7} \mathrm{H}_{13} \mathrm{NH}_{3}\right]_{5}\left[\mathrm{Al}_{3}\left(\mathrm{PO}_{4}\right)_{4}\left(\mathrm{HPO}_{4}\right)\right]$ & $10.118(3)$ & 15.691(4) & $18.117(3)$ & $72.91(2)$ & $85.18(2)$ & $79.49(2)$ & $P(-1)$ & $\begin{array}{l}\text { Alternate three-corner-shared }+ \\
\text { three-edge-shared } 4 \mathrm{R}\end{array}$ & $7 \mathrm{~b}$ \\
\hline$\left[\mathrm{C}_{5} \mathrm{H}_{9} \mathrm{NH}_{3}\right]_{5}\left[\mathrm{Al}_{3}\left(\mathrm{PO}_{4}\right)_{4}\left(\mathrm{HPO}_{4}\right)\right]$ & $10.063(2)$ & $15.447(2)$ & $15.736(2)$ & $71.72(1)$ & $80.07(1)$ & $79.57(1)$ & $P(-1)$ & $\begin{array}{l}\text { Alternate three-corner-shared }+ \\
\text { three-edge-shared }\end{array}$ & $7 \mathrm{a}$ \\
\hline$\left[\left(\mathrm{NH}_{4}\right)\left(\mathrm{C}_{2} \mathrm{~N}_{2} \mathrm{H}_{10}\right)\right]\left[\mathrm{Al}\left(\mathrm{PO}_{4}\right)_{2}\right]$ & $8.033(10)$ & $16.989(2)$ & $8.740(2)$ & 90.0 & 90.0 & 90.0 & Pccn & Corner-shared & $8 \mathrm{a}$ \\
\hline$\left[\mathrm{C}_{2} \mathrm{~N}_{2} \mathrm{H}_{10}\right]\left[\mathrm{Al}\left(\mathrm{PO}_{4}\right)\left(\mathrm{HPO}_{4}\right)\right]$ & $4.901(1)$ & 9.032(1) & 11.691(1) & $81.38(1)$ & $82.27(1)$ & $75.83(1)$ & $P(-1)$ & Edge-shared & $6 \mathrm{~b}$ \\
\hline $\mathrm{Na}_{3}\left[\mathrm{Al}\left(\mathrm{HPO}_{4}\right)\left(\mathrm{PO}_{4}\right)_{2}(\mathrm{OH})\right]$ & $15.277(2)$ & 7.054(9) & $7.040(9)$ & 90.0 & $96.7(2)$ & 90.0 & $C 2 / m$ & Tancoite type & $11 \mathrm{c}$ \\
\hline$\left[\mathrm{C}_{10} \mathrm{~N}_{2} \mathrm{H}_{9}\right]\left[\mathrm{Al}\left(\mathrm{PO}_{4}\right)\left(\mathrm{H}_{2} \mathrm{PO}_{4}\right)\right]$ & $4.9169(9)$ & $10.696(3)$ & $14.660(8)$ & 107.84(3) & $95.68(3)$ & $99.91(2)$ & $P(-1)$ & Edge-shared & $6 \mathrm{a}$ \\
\hline$\left[\mathrm{C}_{3} \mathrm{~N}_{2} \mathrm{H}_{12}\right]\left[\mathrm{Al}\left(\mathrm{HPO}_{4}\right)\left(\mathrm{PO}_{4}\right)\right]$ & $8.309(1)$ & $8.636(1)$ & $8.844(1)$ & $111.9(1)$ & $107.6(1)$ & $98.0(1)$ & $P(-1)$ & Corner-shared & $a$ \\
\hline$\left[\mathrm{C}_{3} \mathrm{~N}_{2} \mathrm{H}_{12}\right]^{2+}\left[\mathrm{Al}\left(\mathrm{HPO}_{4}\right)\left(\mathrm{PO}_{4}\right)\right]$ & $16.832(2)$ & $8.289(3)$ & $8.694(2)$ & 90.0 & 90.0 & 90.0 & Pccn & Corner-shared & $a$ \\
\hline$\left[\left(\mathrm{C}_{3} \mathrm{~N}_{2} \mathrm{H}_{12}\right)\left(\mathrm{N}_{2} \mathrm{O}_{2} \mathrm{H}_{6}\right)_{0.5}\left[\mathrm{Al}\left(\mathrm{PO}_{4}\right)_{2}\right]\right.$ & $8.669(1)$ & $8.943(1)$ & $9.266(1)$ & $98.3(1)$ & $116.0(2)$ & $99.7(1)$ & $P(-1)$ & Corner-shared & $a^{a}$ \\
\hline
\end{tabular}

${ }^{a}$ Present work. 
III, containing organic cations and tetrahedrally coordinated $\mathrm{Al}$ and $\mathrm{P}$ have been prepared under hydrothermal conditions employing the reaction between amine phosphates and $\mathrm{Al}^{\mathrm{III}}$ ions. The chains structure observed in I-III are similar to those in $\left[\mathrm{NH}_{3}\left(\mathrm{CH}_{2}\right)_{4} \mathrm{NH}_{3}\right]\left[\mathrm{Ga}\left(\mathrm{PO}_{4}\right)\right.$ $\left.\left(\mathrm{HPO}_{4}\right)\right],(12),\left[\mathrm{NH}_{3}\left(\mathrm{CH}_{2}\right)_{3} \mathrm{NH}_{3}\right]\left[\mathrm{GaH}\left(\mathrm{PO}_{4}\right)_{2}\right](9 \mathrm{~b})$, and the $\left[\mathrm{AlP}_{2} \mathrm{O}_{8}\right]^{3-}$ chains in $\left[\mathrm{NH}_{4}\right]\left[\mathrm{NH}_{3}\left(\mathrm{CH}_{2}\right)_{2} \mathrm{NH}_{3}\right]$ $\left[\mathrm{Al}\left(\mathrm{PO}_{4}\right)_{2}\right](8 \mathrm{a}),\left[\mathrm{H}_{3} \mathrm{O}\right]\left[\mathrm{NH}_{3}\left(\mathrm{CH}_{2}\right)_{2} \mathrm{NH}_{3}\right]\left[\mathrm{Al}\left(\mathrm{PO}_{4}\right)_{2}\right](9 \mathrm{a})$, and $\left[\mathrm{Et}_{3} \mathrm{NH}\right]\left[\mathrm{Al}_{2}\left(\mathrm{HPO}_{4}\right)_{2}\right](8 \mathrm{~b})$. In Table 10, we list the various one-dimensional aluminum phosphate structures discovered till now. The three new compounds reported in this paper, expands the vast family of structurally diverse aluminum phosphates with the $\mathrm{Al} / \mathrm{P}$ ratio of nonunity (22). These compounds contain $\mathrm{MO}_{4}$ and $\mathrm{PO}_{4}$ tetrahedra alternatively linked to form ribbons of corner-shared four-membered rings. The packing arrangement of the chains and the inter- and intrachain interactions are primarily responsible for the slight variations observed in the structures. Thus in $\left[\mathrm{Et}_{3} \mathrm{NH}\right]\left[\mathrm{Al}_{2}\left(\mathrm{HPO}_{4}\right)_{2}\right](8 \mathrm{~b})$, there are intrachain hydrogen bonding between $\mathrm{P}=\mathrm{O}$ and $\mathrm{P}-\mathrm{OH}$ groups. In the present case, we have interchain hydrogen bonding between $\mathrm{P}=\mathrm{O}$ and $\mathrm{P}-\mathrm{OH}$ units of two different chains (I), as depicted in Fig. 2b. The chain composition of $\mathbf{I}$ is identical to that of $\left[\mathrm{NH}_{3}\left(\mathrm{CH}_{2}\right)_{2} \mathrm{NH}_{3}\right]\left[\mathrm{Al}\left(\mathrm{PO}_{4}\right)\left(\mathrm{HPO}_{4}\right)\right](6 \mathrm{~b})$, but in the latter the structure forms a edge-shared ladder rather than cornershared ones. The amines in I-III differ marginally and the differences in their chain compositions probably result from such minor differences.

Considering that very few linear chain metal phosphates are known, the isolation and characterization of three corner-shared chain AlPOs of different chain compositions is of some value. This is especially because four-membered rings and corner-shared chains are supposed to be the basic building units involved in the formation of open-framework metal phosphates. In the mechanism proposed for the formation of open-framework aluminum phosphates (7a), the corner-shared chain structure has been identified as the primary building unit. Isolation of the linear chain AlPOs appears to be easier with amine phosphate route compared to the conventional hydrothermal method.

\section{ACKNOWLEDGMENTS}

The authors thank Professor C. N. R. Rao, FRS, for his help, support and encouragement. A.A.A. gratefully acknowledges the Third World Academy of Sciences (TWAS) for the award of a South-South fellowship.

\section{REFERENCES}

1. (a) S. T. Wilson, B. M. Lok, and E. M. Flanigen, U.S. patent 4310440 (1982); (b) S. T. Wilson, B. M. Lok, C. A. Messina, T. R. Cannan, and E. M. Flanigen, J. Am. Chem. Soc. 104, 1146 (1982).

2. J. M. Bennett, J. M. Cohen, G. Artiol, J. J. Pluth, and J. V. Smith, Inorg. Chem. 24, 188 (1985).

3. S. Natarajan, J.-C.P. Gabriel, and A. K. Cheetham, J. Chem. Soc. Chem. Commun. 1415 (1996) and references therein.

4. J. M. Thomas, R. H. Jones, R. Xu, J. Chen, A. M. Chippindale, S. Natarajan, and A. K. Cheetham, J. Chem. Soc. Chem. Commun. 929 (1992).

5. A. Choudhury, S. Natarajan, and C. N. R. Rao, Int. J. Inorg. Mater. 2, 87 (2000).

6. (a) A. M. Chippindale and C. Turner, J. Solid State Chem. 128, 318 (1997); (b) I. D. Williams, J. Yu, Q. Gao, J. Chen, and R. Xu, Chem. Commun. 1273 (1997).

7. (a) S. Oliver, A. Kuperman, A. Lough, and G. A. Ozin, Chem. Mater. 8, 2391 (1996); (b) S. Oliver, A. Kuperman, A. Lough, and G. A. Ozin, Inorg. Chem. 35, 6373 (1996).

8. (a) Q. Huo, J. Chen, S. Li, R. Xu, J. M. Thomas, M. Light, and M. B. Hursthouse, J. Solid State Chem. 127, 145 (1996); (b) R. H. Jones, J. M. Thomas, R. Xu, Y. Xu, Q. Huo, A. K. Cheetham, and D. Bieber, J. Chem. Soc. Chem. Commun. 1170 (1990).

9. (a) W. Tieli, Y. Long, and P. Wenqin. J. Solid State Chem. 89, 392 (1990); (b) T. Loisseau, F. Serpaggi, and G. Ferey, Chem. Commun. 1093 (1997).

10. (a) S. Neeraj, S. Natarajan, and C. N. R. Rao, Angew. Chem. Int. Ed.38, 3480 (1999); (b) C. N. R. Rao, S. Natarajan, and S. Neeraj, J. Am. Chem. Soc. 126, 2810 (2000).

11. (a) R. A. Ramik, B. D. Sturman, P. J. Dunn, and A. S. Poverennykj, Can. Mineral. 18, 185 (1980); (b) M. P. Attfield, R. E. Morris, I. Burshtein, C. F. Campana, and A. K. Cheetham, J. Solid State Chem. 118, 412 (1995); (c) K.-H. Lii and S.-L. Wang, J. Solid State Chem. 128, 21 (1997).

12. A. M. Chippindale, A. D. Bond, A. D. Law, and A. R. Cowley, J. Solid State Chem. 136, 227 (1998).

13. S. Kamoun, A. Jouini, and A. Dasud, Acta Crystallogr. C 36, 1481 (1980); see also Acta Crystallogr. C 47, 1481 (1991).

14. "Siemens Users Manual." Siemens Analytical X-ray Instruments, Madison, WI, 1995.

15. G. M. Sheldrick, "SADABS Siemens Area Detector Absorption Correction Program." Univ. of Gottingen, Gottingen, Germany, 1994.

16. G. M. Sheldrick, "SHELXS-86, A Program for the Solution of Crystal Structures." Univ. of Göttingen, Göttingen, Germany, 1986.

17. G. M. Sheldrick, "SHELXTL-PLUS Program for Crystal Structure Solution and Refinement." Univ. of Göttingen, Göttingen, Germany, 1993.

18. C. N. R. Rao, S. Natarajan, and S. Neeraj, J. Solid State Chem. 152, 302 (2000).

19. I. D. Brown and D. Aldermatt, Acta Crystallogr. B 41, 244 (1984).

20. A. M. Chippindale, S. Natarajan, J. M. Thomas, and R. H. Jones, J. Solid State Chem. 111, 18 (1994).

21. B. Dickens, E. Prince, L. W. Schroeder, and T. H. Jorden, Acta Crystallogr. B 30, 1470 (1974).

22. Jihong Yu, Ruren Xu, and Jiyang Li, Solids State Sci. 2, 181 (2000). 